\title{
Quantum effects of thermal conductance through atomic chains
}

\author{
A. Ozpineci and S. Ciraci \\ Department of Physics, Bilkent University, Bilkent 06533, Ankara, Turkey \\ (Received 31 July 2000; revised manuscript received 21 November 2000; published 13 March 2001)
}

\begin{abstract}
We present a formalism for an atomic scale study of phononic heat transfer. The expression of thermal energy current can be cast in the Landauer form and incorporates the transmission coefficient explicitly. Calculation of the thermal conductance of a monoatomic chain of $N$ atoms between two reservoirs shows interesting quantum features. The conductance density appears as Lorentzian type resonances at the eigenfrequencies of the chain. At low-temperature limit the discrete vibrational frequency spectrum of a "soft" chain may reflect on the thermal conductance by giving rise to a sudden increase. At room temperature, the conductance through a "stiff" chain may oscillate with the number of chain atoms. The obtained quantum features are compared with similar effects found in the quantized electrical conductance.
\end{abstract}

DOI: 10.1103/PhysRevB.63.125415

PACS number(s): 63.22. $+\mathrm{m}, 63.20 . \mathrm{Dj}, 66.70 .+\mathrm{f}, 73.23 .-\mathrm{b}$

\section{INTRODUCTION}

The advances in nanofabrication and emerging novel results are now challenging the investigation of very fundamental concepts in quantum physics. Nanowires ${ }^{1}$ even monoatomic chains ${ }^{2}$ suspended between two metal electrodes are produced; their stability, electronic and mechanical properties have become the focus of attention. ${ }^{1-3} \mathrm{Re}-$ cently, the single walled carbon nanotubes, ${ }^{4}$ which can be produced with variety of diameter and chirality have shown very interesting electromechanical properties and dimensionality effects. ${ }^{5}$ These properties have been actively explored with the hope of discovering novel applications in nanotechnology. The ballistic electron transport through an atom or atomic chain have revealed interesting quantum features. ${ }^{1,2,6,7}$ Owing to the finite level spacing of vibrational frequencies the phononic energy transfer through an electrically nonconducting nanoobject (i.e., a molecule, an atomic chain or a single atom) between two reservoirs appears to be an equally interesting subject. Questions one can raise are whether the discrete vibrational frequency spectrum can reflect on the thermal conductance $\mathcal{K}$; what quantum features similar to those of ballistic electron conduction would be involved in the phononic energy transfer through a small molecule or wire. Beyond being an academical interest, providing satisfactory answers to these questions is essential for several physical events and chemical processes in biology, molecular electronics, and nanoscience.

The phononic energy transfer through dielectric wires have been subject of recent interest. ${ }^{8-10}$ While wires with relatively larger cross section have been treated within the continuum approach, ${ }^{8,9}$ the heat transfer through a nanoobject or a monoatomic chain presents interesting features and complexities owing to atomic scale contacts with the heat reservoirs. In this study, we develop a formalism for an atomic scale study of phononic heat transfer. It is based on the Keldysh's theory of non-equilibrium processes. ${ }^{11}$ The Keldysh's theory was used earlier to investigate the ballistic electron conduction through a point contact. ${ }^{12}$ Our work presents the formalism for bosons, which is applied for the thermal conductance. We obtained an expression for the thermal energy current $J$ which can be cast in the Landauer form. Our numerical calculations on a finite atomic chain comprising $N$ atoms $(N=1-16)$ between two electrodes (or reservoirs) reveal interesting quantum features, such as resonances and step behavior of thermal conductance, and clarify issues on the variation of $\mathcal{K}$ with $N$ and temperature $T$. Our results are of particular interest for the energy transfer through semiconducting carbon nanotubes and molecules coupled to two reservoirs. In Sec. II, we present the description of our model of phononic heat transfer through an atomic chain and the formalism of heat current and conductance developed by using Keldysh's theory of nonequilibrium processes. The results of numerical calculations and various quantum effects revealed therefrom are discussed in Sec. III.

\section{MODEL AND METHOD}

The physical system of interest is schematically described as an inset in Fig. 1(a). Two reservoirs ( $L$ and $R$ ) with temperatures $T_{L}$ and $T_{R}$ are described by the vibrational Hamiltonians $\mathcal{H}_{L}$ and $\mathcal{H}_{R}$, respectively. $L$ and $R$ are connected by a dielectric chain of $N$ atoms, that is described by the following Hamiltonian:

$$
\mathcal{H}_{S}=\sum_{i=1}^{N} \frac{p_{i}^{2}}{2 M}+\sum_{i=1}^{N-1} \frac{k}{2}\left(x_{i}-x_{i+1}\right)^{2}+\frac{k}{2} x_{1}^{2}+\frac{k}{2} x_{N}^{2},
$$

where $p_{i}$ and $x_{i}$ are the momentum and displacement of the $i$ th atom, respectively. In this Hamiltonian the coupling to $L$ and $R$ is not included, and in the chain only the harmonic interactions with the nearest neighbor coupling $k$, are taken into account. The end atoms $(i=1$ and $i=N)$ are connected to the surfaces of the reservoirs. The coupling of the chain to $L$ and $R$ is described at the lowest order by the Hamiltonian

$$
\mathcal{H}_{\text {int }}=A_{L} u_{L} x_{1}+A_{R} u_{R} x_{N},
$$

where $u$ 's stands for the lateral displacements of reservoir atoms which are coupled with the chain. $A_{L}$ and $A_{R}$ are coupling parameters. It is assumed that only one atom from each reservoir interacts with the chain and only the longitudinal modes are considered. Using this simple model we aim to reveal the underlying physics of phononic energy transfer through atomic wires. Nevertheless, generalization to con- 

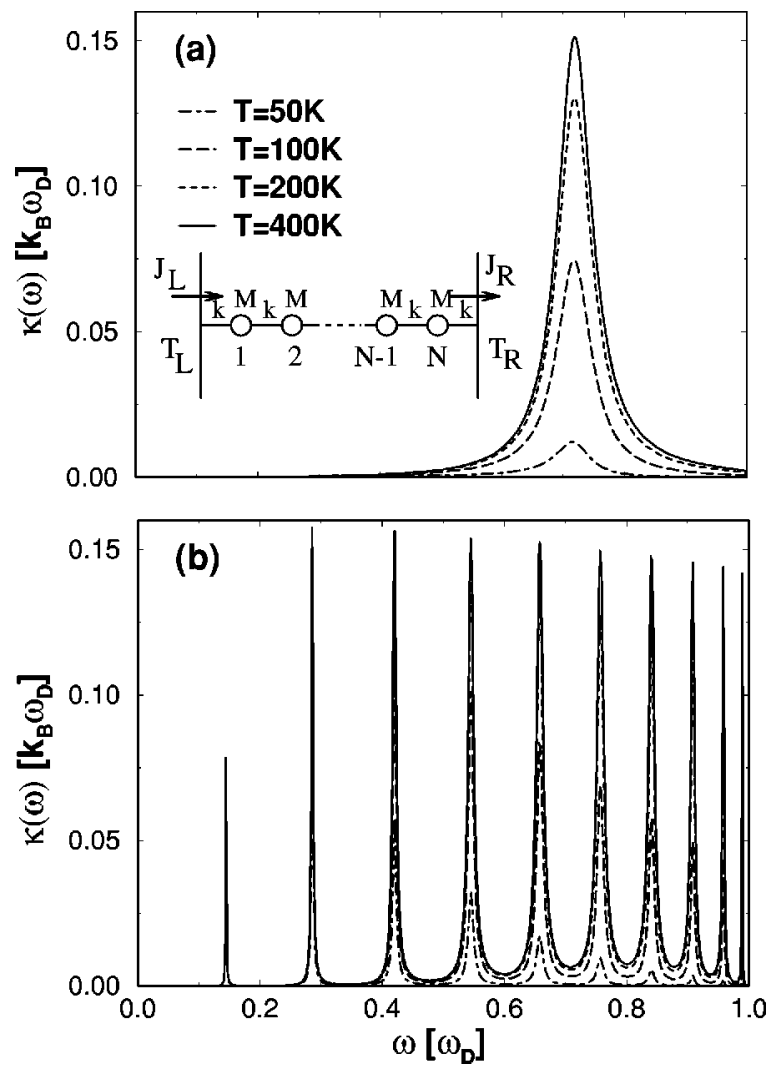

FIG. 1. Variation of the conductance density $\mathcal{K}(\omega)$ with temperature $T$, and number of atoms in the chain $N$, for $\sqrt{k / M}$ $=0.5 \omega_{D}$. The inset describes the model used for the phononic energy transfer. The chain consists of $N$ atoms; each atom of mass $M$ is under the harmonic interaction with nearest neighbor coupling $k$. (a) $N=1$; (b) $N=10$.

sider the coupling between distant atoms beyond the nearest neighbor interaction and to include transversal modes is possible. Even the first-principles calculation of phonon spectrum for a specific sample can be provided. While these sample specific complex models require tremendous computational efforts, their conclusions concerning the objective of this study would not change in any essential manner as long as the eigenfrequencies of the atomic chain have finite level spacings. Since $T_{L}$ and $T_{R}$ are kept constant and $T_{L}>T_{R}$, the system is not in equilibrium, but in steady state. Then the phononic energy transfer from $L$ to $R$ can conveniently be described by the Keldysh's theory. ${ }^{11}$

A few comments about the application of the Keldysh's theory are in order; for a more detailed discussion, see Ref. 11, eg. Within the Keldysh formalism, the expectation value of any operator $\mathcal{O}$ in steady state can be expressed as, $\langle\mathcal{O}\rangle$ $=\left\langle\mathcal{T}_{\mathcal{C}} \mathcal{O}\left(0^{+}\right) e^{-(i / \hbar) \int_{\mathcal{C}} H_{\text {int }}(t) d t}\right\rangle_{\text {connected }}$ by using the path ordering operator, $\mathcal{T}_{C}$, and summing only connected Feynman diagrams. In terms of the two-point correlation function of the displacement of the atoms $\mathcal{G}_{i j}^{\zeta_{1} \zeta_{2}}\left(t-t^{\prime}\right)$ $=\left\langle\mathcal{T}_{C} x_{i}\left(t^{\zeta_{1}}\right) x_{j}\left(t^{\prime} \zeta_{2}\right)\right\rangle$ with $\zeta_{i}=+$ or - , one defines the following Green's function matrices and self-energy matrices:

$$
\mathcal{G}_{i j}\left(t-t^{\prime}\right)=\left(\begin{array}{ll}
\mathcal{G}_{i j}^{++}\left(t-t^{\prime}\right) & \mathcal{G}_{i j}^{+-}\left(t-t^{\prime}\right) \\
\mathcal{G}_{i j}^{-+}\left(t-t^{\prime}\right) & \mathcal{G}_{i j}^{--}\left(t-t^{\prime}\right)
\end{array}\right),
$$

$$
\Sigma_{i j}\left(t-t^{\prime}\right)=\left(\begin{array}{cc}
\Sigma_{i j}^{++}\left(t-t^{\prime}\right) & \Sigma_{i j}^{+-}\left(t-t^{\prime}\right) \\
\Sigma_{i j}^{+}\left(t-t^{\prime}\right) & \Sigma_{i j}^{-}\left(t-t^{\prime}\right)
\end{array}\right) .
$$

In terms of the Fourier transformed Green's function matrices, the Dyson's equation takes the form $\mathcal{G}_{i j}(\omega)={ }^{0} \mathcal{G}_{i j}(\omega)$ $+\sum_{j^{\prime} k^{\prime}} \mathcal{G}_{i j^{\prime}}(\omega) \Sigma_{j^{\prime} k^{\prime}}(\omega) \mathcal{G}_{k^{\prime} j}(\omega)$. Note that these Green's functions are linearly dependent. ${ }^{13}$ Using the linear dependence, the matrices $\mathcal{G}_{i j}$ and $\Sigma_{i j}$ can be transformed and the Dyson equation for advanced $\mathcal{G}^{A}$, retarded $\mathcal{G}^{R}$, and Keldysh $\mathcal{G}^{K}$, Green's functions are written. ${ }^{14}$

We note that the operator corresponding to the current at the contact can be obtained from the continuity equation written in the form, $d \epsilon / d t=-\left(J_{R}-J_{L}\right)$, where $\epsilon$ is the total energy operator of the wire. The operators corresponding to the heat current leaving $L$ and entering in $R$, respectively, can be expressed as $J_{L}=-\left(A_{L} / M\right) u_{L} p_{1}$ and $J_{R}$ $=\left(A_{R} / M\right) u_{R} p_{N}$. Then the expectation value of the current through the wire is

$$
J=\left\langle J_{L}\right\rangle=-\left(A_{L} / M\right)\left\langle u_{L}(t) p_{1}(t)\right\rangle
$$

which can be expressed as

$$
J=-A_{L} \int_{-\infty}^{\infty} \frac{d \omega}{2 \pi}(-i \omega)\left\langle u_{L}(t) x_{i}(t)\right\rangle_{\omega},
$$

where

$$
\left\langle u_{L}(t) x_{i}\left(t^{\prime}\right)\right\rangle=\int \frac{d \omega}{2 \pi} e^{i \omega\left(t-t^{\prime}\right)}\left\langle u_{L}(t) x_{i}\left(t^{\prime}\right)\right\rangle_{\omega} .
$$

By using the Wick's theorem Eq. (5) can be written as

$$
\begin{aligned}
J= & -\sum_{\alpha=1, N} \int \frac{d \omega}{2 \pi}(\hbar \omega)\left[\Sigma_{1 \alpha}^{++}(\omega) \mathcal{G}_{\alpha 1}^{++}(\omega)\right. \\
& \left.+\Sigma_{1 \alpha}^{+-}(\omega) \mathcal{G}_{\alpha 1}^{-+}(\omega)\right] .
\end{aligned}
$$

In the present study, the summation sign in Eq. (7) involves only $\alpha=1$ and $\alpha=N$ terms since in our model we have just these two contacts. In the general case one should sum over all contacts. In our model, where $\mathcal{H}_{\text {int }}$ is given by Eq. (2), the self-energy can be calculated exactly: $\Sigma_{i j}(\omega)$ $=\Sigma_{11}(\omega) \delta_{i 1} \delta_{j 1}+\Sigma_{N N}(\omega) \delta_{i N} \delta_{j N}$, where $\quad \Sigma_{i i}\left(t-t^{\prime}\right)$ $=\left\langle\mathcal{T}_{C} u_{i}(t) u_{i}\left(t^{\prime}\right)\right\rangle_{0}$. Finally, substituting $\Sigma_{i j}(\omega)$ into the expression of Dyson equation, the current expression can be cast in the following form;

$$
J=\frac{1}{2 \pi} \sum_{m l} \int d \omega \hbar \omega\left[n^{L}(\omega)-n^{R}(\omega)\right] T_{m l}(\omega),
$$

that has the Landauer form. ${ }^{15} n^{L}(\omega, T)$ and $n^{R}(\omega, T)$ are Bose-Einstein distribution functions for $L$ and $R$, respectively. $T_{m l}(\omega)$ stands for the transmission coefficient for a phonon of frequency $\omega$ at the $m$ th branch of $L$ to the $l$ th branch of $R$, and is expressed as 


$$
\begin{aligned}
T_{m l}(\omega)= & (2 \pi)^{2}\left(\frac{A_{L} A_{R}}{\hbar^{2}}\right)^{2} g_{m}^{L}(\omega) g_{l}^{R}(\omega) \operatorname{det} \mathcal{G}_{1 N}(\omega) \\
& \times \frac{\hbar}{2 M_{L} \omega} \frac{\hbar}{2 M_{R} \omega},
\end{aligned}
$$

where $\operatorname{det} \mathcal{G}_{1 N}=\mathcal{G}_{1 N}^{A} \mathcal{G}_{1 N}^{R} \cdot g_{m}^{L(R)}$ and $M_{L(R)}$ are the density of states corresponding to $m$ th branch and mass of atoms of $L(R)$, respectively.

Next we apply the above formalism to investigate the phononic heat transfer and related thermal conductance through a finite, monoatomic chain between two reservoirs as schematically presented in Fig. 1(a). We assume that both reservoirs are identical and their phonon densities of states are treated within the Debye approximation. We take $\hbar \omega_{D}^{R}$ $=\hbar \omega_{D}^{L}=\hbar \omega_{D}=37.6 \mathrm{meV} ; M_{L}=M_{R}=56 \mathrm{amu}, M=28 \mathrm{amu}$; $A_{L}=A_{R}=-19 \mathrm{~J} / \mathrm{m}^{2}$. Also the heat current from $\mathrm{L}$ to $\mathrm{R}$ at steady state is, $J\left(T_{L}, T_{R}\right)=\int_{0}^{1} d x J\left(x \omega_{D}, T_{L}, T_{R}\right)$, where $J(\omega, T)$ is the heat current density at the frequency $\omega$. Then the heat conductance density at $T$ is defined by $\mathcal{K}(\omega)$ $=\lim _{\Delta T \rightarrow 0} J(\omega, T+\Delta T, T) / \Delta T$, so that the total conductance is obtained by the integral $\mathcal{K}=\int_{0}^{1} d x \mathcal{K}\left(x \omega_{D}, T\right)$.

\section{RESULTS AND DISCUSSION}

In this section, we discuss the results obtained from the model described in the previous section. The numerical results illustrated in Figs. 1-4 reveal novel features and interesting quantum effects in atomic scale heat transfer. Figures $1(a), 1(b)$ shows the variation of the conductance density $\mathcal{K}(\omega)$, with temperature and number of atoms in the chain $N$. $\mathcal{K}(\omega)$ appears as Lorentzian type resonances at the eigenfrequencies of the chain, $\omega_{i}$. The height of resonances depends on $T$ and $\omega_{i}$. The higher $\omega_{i}$ and the lower $T$, the lower is the height of resonances. This behavior originates from the Bose-Einstein distribution function. At sufficiently high temperatures, the heights of the resonances become independent of $N$. However, $\mathcal{K}(\omega)$ resonances become narrow as $N$ becomes large. This can be understood in terms of the weakening of the coupling constant of each mode which is proportional to $1 / \sqrt{N+1}$.

A system analogous to the present model has been realized in the electrical conductance through a constriction. ${ }^{16}$ Similar to the central atomic chain in the present model, an impurity atom was placed or a double barrier resonant tunneling (DBRT) structure was formed in the constriction connecting two 2D electron gas reservoirs. The quantized energy levels of the impurity atom or the DBRT structure with finite level spacing has reflected on the transport of electrons yielding periodic oscillations as a function of the gate voltage or equally chemical potential. ${ }^{16}$ The behavior of the observed conductance is reminiscent of the phononic conductance described in Fig. 1, except the temperature dependence of the heights of resonances (or peaks) and their periodic oscillations. The periodic oscillations were explained in terms of single-electron charging. ${ }^{17}$ In the absence of the Coulomb interaction energy the resonances occur at the quantized energies $^{18}$ as in Fig. 1. The anomalous temperature depen- dence of the resonances, i.e., the irregular and nonmonotic dependence of the heights of resonances on temperature, in contrast to the $1 / T$ dependence of the Fermi distribution, is explained in terms of the participation of multiple electronic levels in each resonance. ${ }^{19}$

The dependence of the total conductance $\mathcal{K}$ on the material parameters, $k$ and $M$ through $\Omega=\sqrt{k / M}$ is illustrated in Figs. 2(a), 2(b). As $\Omega$ increases, the eigenfrequencies of the modes, $\omega_{i}$, increase; starting from the highest one, they cross the Debye frequency, $\omega_{D}$, one by one. Each $\omega_{i}$ rised above $\omega_{D}$, and thus came out of the range of $g^{L(R)}(\omega)$ ceases to contribute to the thermal conductance. This way, a channel is closed and $\mathcal{K}$ is decreased suddenly leading to a step structure in the variation of $\mathcal{K}$ with $\Omega$. The larger the level spacing $\Delta \omega_{i}$, the longer becomes the plateaus. Since all $\omega_{i}<\omega_{D}$ contribute to the thermal conduction at high temperature, one can obtain several step structure for large $N$ $(N>3)$. Also the step structure becomes pronounced at high temperature. This situation is contrary to the electronic counterpart; ballistic electron conductance, where the steps become less pronounced due to the smearing of Fermi distribution. ${ }^{6}$ We also note that (i) the step behavior of electrical conductance is obtained by changing the width of the constriction or by stretching the metallic wire between two electrodes. In principle, the step behavior of the ballistic electrical conductance could have also been achieved by changing the Fermi energy $E_{F}$ (or chemical potential $\mu$ at finite temperature). In the present case, the step behavior of $\mathcal{K}$ can be realized to some extent by varying $k$ and $M$, and also $\omega_{D}$. Of course $\omega_{D}$ is an artificial cutoff due to the Debye model. In real crystal, the cutoff of $\omega(\mathbf{k})$ at the zone boundary has to be taken into account. Cutoff frequency can be modified by applying strong external pressure or the eigenfrequencies $\omega_{i}$, can be changed by stretching the chain. According to present results, the value of $\mathcal{K}$ is changed by replacing chain atoms with their isotopes. (ii) The step structure shown in Fig. 2 is modified if there is surface phonons in the gap. (iii) In calculating the step structure, the broadening of the modes, which normally smears the sharp structure, is taken into account. This smearing is more pronounced for the first several steps, since the higher eigenmodes are more closely spaced, and hence they overlap due to broadening. (iv) The Debye density of states has a very sharp cutoff at the Debye frequency. Therefore, as the resonances passes the Debye frequency due to the variation of $\Omega$, their contribution to the conductance ceases abruptly. In a more realistic density of states of crystals, the closing of the channel would be gradual resulting in the smearing of the steps. However, it is expected that the Van Hove singularities are reflected on $\mathcal{K}$ versus $\Omega$ curve. (v) The anharmonic coupling which is not taken into account here, may modify the step behavior especially for very large $\mathrm{N}$, and for $\omega_{i}>\omega_{D}$.

The variation of the total conductance with temperature is shown in Fig. 3 for a particular chain parameter $\Omega$ and $N$ $=5$. At the high-temperature limit, $\left[n^{L}(\omega)-n^{R}(\omega)\right]$ in Eq. (8) can be approximated by $k_{B} \Delta T / \hbar \omega$. Then $\mathcal{K}$ saturates at a value that depends on the couplings $A_{R}$ and $A_{L}$ as well as on the density of states of the reservoirs $L$ and $R$. At low- 

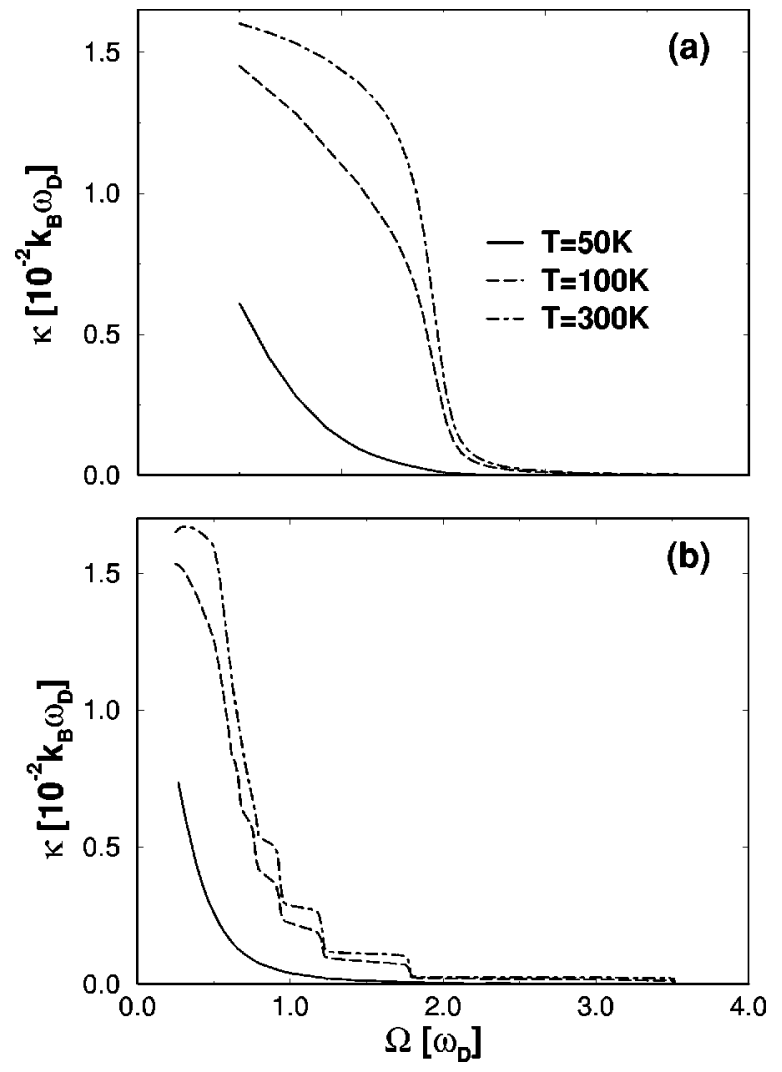

FIG. 2. The variation of the total conductance $\mathcal{K}$ with chain parameters $\Omega=\sqrt{k / M}$. (a) $N=1$; (b) $N=10$.

temperature limit, one expects that the discrete vibrational spectrum can reflect on the variation of $\mathcal{K}$ with temperature, if the wire has a vibrational mode in the range of $k_{B} T$. This situation examined by considering a "soft" chain with $\Omega$ $=\omega_{D} / 100$. The calculated $\mathcal{K}$ versus $T$ is illustrated as an inset to Fig. 3. The sudden increase of $\mathcal{K}$ when the second vibrational mode begins to contribute to the conduction is clearly seen. The present result justifies the similar jumps in the $\mathcal{K}$ versus $T$ curve obtained by using a phenomenological approach. $^{10}$

In Fig. 4, the variation of the total conductance $\mathcal{K}$ with $\mathrm{N}$ for $\Omega=\omega_{D} / 2$ and $\Omega=\omega_{D}$ displays an interesting behavior. For $\Omega=\omega_{D} / 2$ and $T=300 \mathrm{~K}, \mathcal{K}$ becomes independent of the number of atoms in the chain. Only for $N \leqslant 2, \mathcal{K}$ is slightly decreased, since the conductance density $\mathcal{K}(\omega)$ is broadened and it does not contribute to conductance when its tail exceeds $\omega_{D}$. Note that if all the eigenfrequencies, $\omega_{i}$, are smaller than $\omega_{D}, \mathcal{K}$ becomes independent of $N$ at high temperature, despite the number of modes of the chain and hence number of the conductance channels increase with $N$. This situation can be explained in terms of the weakening of the coupling of each mode to the modes of reservoirs with increasing $N$. On the other hand, $\mathcal{K}$ fluctuates for a "stiff", chain with $\Omega=\omega_{D}$. These two different behaviors (corresponding to the cases, $\Omega=\omega_{D / 2}$ and $\Omega=\omega_{D}$ can be explained by the fact that all the modes contribute to the conductance in the former case, since $\omega_{D}>\omega_{i}$. Whereas, in the latter case, $\omega_{D}$ lies within the spectrum of the wire and the number of contributing modes changes with $N$. The fluctua-

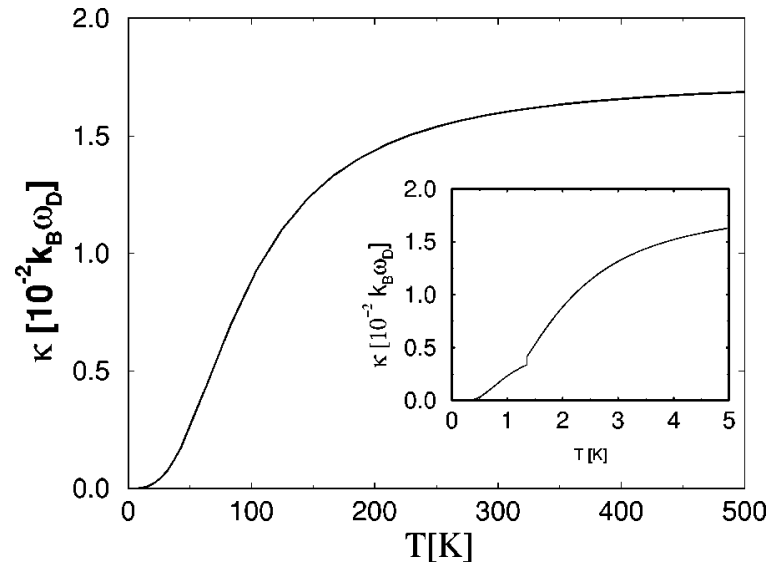

FIG. 3. The total conductance $\mathcal{K}$ versus temperature for $N=5$ and $\sqrt{k / M}=0.5 \omega_{D}$.

tions diminish with increasing $N$ and decreasing $T$. Note that, since the low temperature structure of the conductance versus temperature curve depends on the positions of the eigenmodes which in turn depend on the number of atoms, conductance might show slight dependence on $N$ at low temperatures.

In conclusion, we developed a formalism to calculate the phononic heat transfer through an atomic chain between two reservoirs. The expression of the thermal energy current incorporates the contribution of tunneling and ballistic phonon transfers. We showed that the finite level spacings of the eigenfrequencies of an atomic chain (or nanoobject) reflect on the variation of the phononic thermal conductance. Resonance structure is revealed in the variation of conductance density. One channel of heat conduction is closed as soon as an eigenfrequency of the chain comes out of the range of quasi-continuous phonon frequency (or density of states) of reservoirs. This gives rise to an effect analogous to the formation of plateaus observed in the electrical conductance of a quasi-1D constriction with variable width or of a stretching metal wire. At high temperature limit, the conductance saturates at a value that depends on the material properties of the reservoirs as well as on their couplings with the chain. At room temperature, the conductance through a stiff chain may oscillate with the number of atoms $N$.

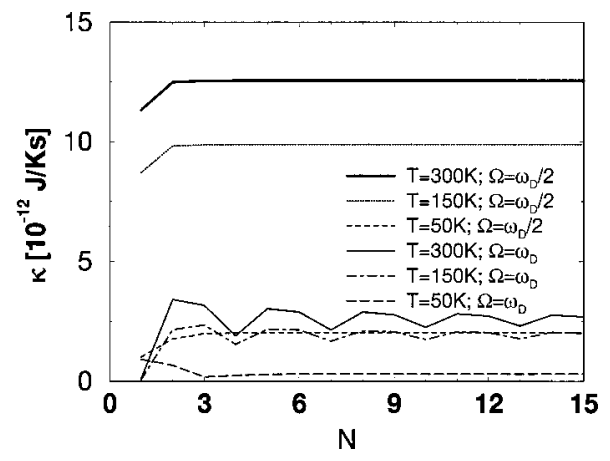

FIG. 4. The dependence of the total conductance on the number of atoms at various temperatures for two different chain parameters $\Omega=\sqrt{k / M}=\omega_{D}$ and $\Omega=\sqrt{k / M}=0.5 \omega_{D}$. 
${ }^{1}$ N. Agraït, J. G. Rodrigo, and S. Vieira, Phys. Rev. B 47, 12345 (1993); J. Pascual, J. I. Mendez, J. G. Herrero, A. M. Baro, N. Garcia, U. Landman, W. D. Luedke, E. N. Bogachek, and H. P. Cheng, Science 267, 1793 (1995); J. M. Krans, C. J. Müller, I. K. Yanson, T. C. M. Gowaent, R. Hesper, and J. M. Ruitenbeek, Phys. Rev. B 48, 14721 (1994).

${ }^{2}$ H. Ohnishi, Y. Kondo, and K. Takayanagi, Nature (London) 395, 780 (1998); A. I. Yanson, G. R. Bolliger, H. E. Brom, N. Agraït, and J. M. Ruitenbeek, ibid. 395, 783 (1998).

${ }^{3}$ D. S. Portal, E. Artacho, J. Junquera, P. Ordejon, A. Garcia, and J. M. Soler, Phys. Rev. Lett. 83, 3884 (1999); J. A. Torres, E. Tosatti, A. Dal Corso, F. Ercolessi, J. J. Kohanoff, F. D. Di Tolla, and J. M. Soler, Surf. Sci. Lett. 426, L441 (1999).

${ }^{4}$ S. Iijima, Nature (London) 354, 56 (1991).

${ }^{5}$ J. W. G. Wildöer, L. G. Venema, A. G. Rinzler, R. E. Smalley, and C. Dekker, Nature (London) 391, 59 (1998); T. W. Odom, J. L. Huang, P. Kim, and C. M. Lieber, ibid. 391, 62 (1998).

${ }^{6}$ S. Ciraci and E. Tekman, Phys. Rev. B 40, R11 969 (1989); E. Tekman and S. Ciraci, ibid. 39, 8772 (1989); 43, 7145 (1991); H. Mehrez and S. Ciraci, ibid. 56, 12632 (1997); H. Mehrez, S. Ciraci, A. Buldum, and I. P. Batra, ibid. 55, R1981 (1997).

${ }^{7}$ A. Yazdani, D. M. Eiglers, and N. D. Lang, Science 272, 1921 (1996); N. D. Lang, Phys. Rev. Lett. 79, 1357 (1997).

${ }^{8}$ D. E. Angelescu, M. C. Cross, and M. L. Roukes, Superlattices
Microstruct. 23, 673 (1998).

${ }^{9}$ L. G. C. Rego and G. Kirczenow, Phys. Rev. Lett. 81, 232 (1998).

${ }^{10}$ A. Buldum, S. Ciraci, and C. Y. Fong, J. Phys.: Condens. Matter 12, 3349 (2000); A. Buldum, D. M. Leitner, and S. Ciraci, Europhys. Lett. 47, 208 (1999). In these papers, an approach based on the chemical rate theories was used.

${ }^{11}$ L. V. Keldysh, Sov. Phys. JETP 20, 1018 (1965).

${ }^{12}$ J. Ferrer, A. M-Rodero, and F. F. Flores, Phys. Rev. B 38, 10113 (1988); later, H. M. Pastawski, [ibid. 46, 4053 (1992)] presented an extensive theory for fermions.

${ }^{13}$ The Green's functions satisfy the following relation: $\mathcal{G}^{++}$ $+\mathcal{G}^{--}-\mathcal{G}^{+-}-\mathcal{G}^{-+}=0$ and $\Sigma^{++}+\Sigma^{--}+\Sigma^{+-}+\Sigma^{-+}=0$.

${ }^{14}$ Note that $\mathcal{G}^{R}=\mathcal{G}^{++}-\mathcal{G}^{-+}, \mathcal{G}^{A}=\mathcal{G}^{++}-\mathcal{G}^{+-}$, and $\mathcal{G}^{K}=\mathcal{G}^{+-}$ $+\mathcal{G}^{-+}$.

${ }^{15}$ R. Landauer, Philos. Mag. 21, 863 (1970); see also D. C. Langreth and E. Abrahams, Phys. Rev. B 24, 2978 (1981).

${ }^{16}$ H. H. F. Scott-Thomas, S. B. Field, M. A. Koster, H. I. Smith, and D. A. Antoniadis, Phys. Rev. Lett. 62, 583 (1989).

${ }^{17}$ H. van Houten and C. W. J. Beenakker, Phys. Rev. Lett. 63, 1893 (1989).

${ }^{18}$ E. Tekman and S. Ciraci, Phys. Rev. B 40, R10 286 (1989); 42, 9098 (1990).

${ }^{19}$ Y. Meir, N. S. Wingreen, and P. A. Lee, Phys. Rev. Lett. 66, 3038 (1991). 